\title{
An array of highly selective Fabry-Perot optical channels for biological fluid analysis by optical absorption using a white light source for illumination
}

\author{
G Minas $^{1}$, R F Wolffenbuttel ${ }^{2}$ and J H Correia $^{1}$ \\ ${ }^{1}$ Department of Industrial Electronics, University of Minho, Campus de Azurém, \\ 4800-058 Guimarães, Portugal \\ ${ }^{2}$ Department ME, Faculty of EEMCS, Delft University of Technology, Mekelweg 4, \\ 2628 CD Delft, The Netherlands \\ E-mail: gminas@dei.uminho.pt
}

Received 13 September 2005, accepted for publication 19 January 2006 Published 14 February 2006

Online at stacks.iop.org/JOptA/8/272

\begin{abstract}
This paper describes a laboratory microsystem (Microlab) used to measure the concentration of biomolecules in biological fluids. Rather than just one measurement channel, it comprises 16 optical channels that enable the measurement of the concentration of 16 different biomolecules with the same device. An array of 16 optical filters based on Fabry-Perot thin-film optical resonators has been designed. Each optical channel is sensitive in a single wavelength with a FWHM less than $6 \mathrm{~nm}$ and with a peak intensity higher than $86 \%$. The 16 optical channel array fabrication requires only four masks, used with different deposition time. The Microlab can easily be tuned during fabrication to analyse different biomolecules only by adjusting the deposition times without affecting the device layout. A commercially available passband optical filter with a passband wavelength in the range $450-650 \mathrm{~nm}$ is used. The Microlab requires only a white light source for illumination due to the use of selective optical filters. The quantitative measurement of uric acid in urine is demonstrated. Such a device is extremely suitable for clinical diagnosis application in clinical laboratories and at a patient's home because of its small size, low cost and portability.
\end{abstract}

Keywords: optical filter array, Fabry-Perot, optical absorption, biomolecules analysis

(Some figures in this article are in colour only in the electronic version)

\section{Introduction}

For disease prevention, diagnostic and treatment patients are often subjected to analyses of their biological body fluids. In clinical diagnostics, the spectrophotometry by optical absorption based on colorimetric detection is the most commonly used analytical technique for determining the concentration and/or amount of a particular biomolecule in biological fluid samples. Usually, those analyses are carried out in clinical laboratories and the results become available after several hours, sometimes days. As a consequence a reliable diagnosis cannot be performed within the consultation time. Mistakes in the logistics, such as lost samples and mislabelling, may further delay the diagnosis [1]. The optical absorption spectrophotometric automated equipment used in a state-of-the-art laboratory reduces errors, but it uses high sample and reagent volumes, making the analysis systems expensive, and does not contribute to patient 
comfort [2]. Outside the laboratory environment, reagent strips for colorimetric detection are commercially available [3]. Such strips are intended for a limited set of biomolecules to be analysed and provide a qualitative result in the form of a colour readout. The need for rapid and on-line measurements with low sample volumes has led to the development of microsystems with the fluidic, detection and readout systems integrated in a single module. Previously developed laboratory microsystems in a single module with absorbance detection require waveguides inserted into the device or a wavelengthdependent light for illumination $[4,5]$. Illumination using only a white light source requires the use of selective optical filters.

This paper describes a laboratory microsystem (Microlab) for the spectrophotometric analysis of biological fluids, especially for colorimetric measuring, by optical absorption, the concentration of 16 biomolecules in those fluids. It comprises an array of 16 highly selective optical filters, which make it portable and ensure that the analysis can be performed at any location with instantaneous results at low cost. This device should have the same reliability, precision and sensitivity as the spectrophotometric automated equipment used in clinical analysis laboratories and, at the same time, it should feature the portability, cost and patient comfort of the reagent strips. In addition, the optical absorption technique is the one that better features these requirements at low cost.

\section{Background of spectrophotometric analysis}

Spectrophotometric analysis is based on colorimetric detection by the optical absorption in a part of the visible spectrum defined by the reaction of the specific biomolecules with a specific reagent. This reaction has an absorption maximum at a specific wavelength. The absorbance value at this wavelength is directly proportional to the concentration of those biomolecules in the samples. However, many of the analytes (the substance being analysed, e.g., element, ion, compound or molecule) of interest for clinical analysis do not have chromophores that absorb light in a useful part of the visible range. Specific chemical reactions are available (reagents) to transform these analytes into coloured products that do have adequate absorbance [6]. In addition, the biomolecule concentration is measured by using a mixture of a reagent with the sample. The measurement method has the following characteristics:

(1) the intensity of the colour produced by the mixture is directly proportional to the biomolecule concentration;

(2) the absorption spectra of the mixture show a maximum peak at a specific wavelength;

(3) each mixture presents a linear behaviour within the concentration range of interest [7].

\section{Design of the 16 optical channel array}

A microsystem to measure the concentration of biomolecules in biological fluids, by optical absorption, was previously implemented [5]. Its operation was successfully demonstrated in uric acid concentration detection. However, the measurements were carried out with a wavelength-dependent light source (monochromatic light).

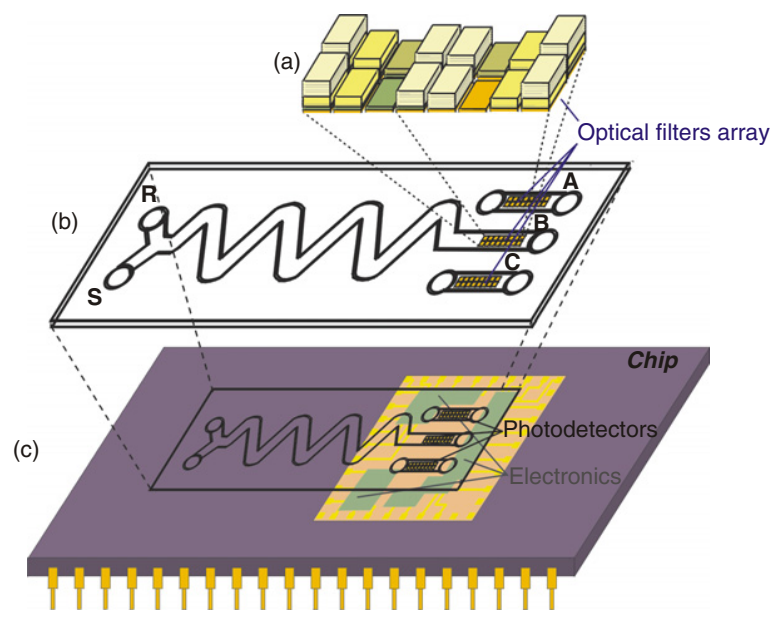

Figure 1. Artist's impression of the laboratory microsystem (Microlab).

\subsection{Complete structure}

An optical passband filter on top of the Microlab allows the use of only a white light source for illumination, which avoids the use of a wavelength-dependent light source, like a monochromatic light or a laser. This characteristic makes the Microlab portable and ensures that the analysis can be performed at any location with instantaneous results, without the use of complex and expensive analysis systems. Figure 1 shows an artist's impression of the Microlab. It combines in a multichip module the optical filters (see figure 1(a)), the microchannels (see figure 1(b)), the photodetectors and the readout electronics (see figure $1(\mathrm{c})$ ).

The microchannels' die (see figure 1(b)) comprises a mixing main channel, for the mixing of the reagent, $\mathbf{R}$, with the sample, $\mathbf{S}$, and three detection microchannels, $\mathbf{A}, \mathbf{B}$, and $\mathbf{C}$. Microchannel A contains the reagent (without biomolecules) and it is needed to obtain the baseline reference and to calibrate the light source. Microchannel $\mathbf{B}$ allows the mixed solution analysis; it contains the reagent plus the sample with biomolecules. Microchannel $\mathbf{C}$ is needed to calibrate the biomolecule concentration (with a well-known concentration standard) and also to compensate the white light oscillations. The three microchannels use the same set of optical filters for proper calibration. For measurements carried out with a wavelength-dependent light source, only two detection microchannels would be needed [5]. All microchannels are $1 \mathrm{~mm}$ wide and $500 \mu \mathrm{m}$ deep. This depth (the optical pathlength) is crucial to achieve measurable absorption with improved sensitivity (the intensity of the measured signal is proportional to the detection volume).

The Microlab operation is based on optical absorption in a well-defined wavelength of the visible spectrum. A white light beam is guided through the microchannels (A, B and $\mathbf{C}$ ) containing the samples to analyse (see figure 2 ). The impinging light is filtered by the optical filters, to a narrow spectral band centred at the wavelength for which the coloured mixture being analysed has its absorption maximum. The intensity of the selected spectral component transmitted through the fluid is measured using underlying photodetectors, vertically aligned with the optical filters. This optical intensity is proportional to 


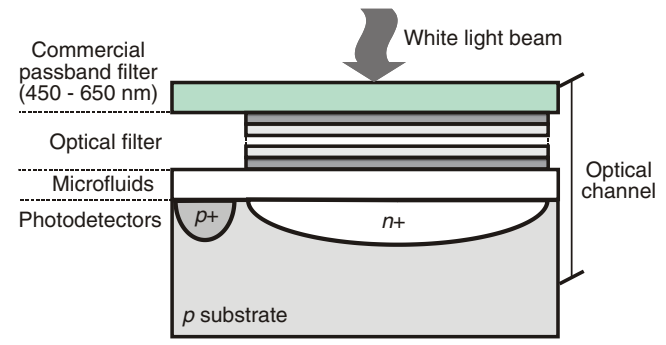

Figure 2. Cross-section of the Microlab structure for a single optical channel.

Table 1. The 16 biomolecules that can be analysed in the Microlab [6]. (Note: U: urine, S: serum, P: plasma, CSF: cerebrospinal fluid. Some of the coloured mixtures have not only a single wavelength for their maximum of absorbance, but a wavelength range [6]. In that case, the wavelength that simplifies the filter fabrication has been selected.)

\begin{tabular}{lll}
\hline Biomolecule & Biological fluid & $\begin{array}{l}\text { Absorption spectra } \\
\text { maximum peak (nm) }\end{array}$ \\
\hline 17-Ketosteriods & $\mathrm{U}$ & 480 \\
Chlorine & $\mathrm{U}, \mathrm{S}$ & 488 \\
Uric acid & $\mathrm{U}, \mathrm{CSF}$ & 495 \\
Cholesterol & $\mathrm{U}, \mathrm{S}$ & 503 \\
Glucose & $\mathrm{U}, \mathrm{S}$ & 512 \\
Magnesium & $\mathrm{U}, \mathrm{S}$ & 520 \\
Creatinine & $\mathrm{U}, \mathrm{S}, \mathrm{P}$ & 528 \\
Urea & $\mathrm{U}$ & 536 \\
Hemoglobin & $\mathrm{U}, \mathrm{P}$ & 544 \\
$\beta$ glucuronidase & $\mathrm{U}, \mathrm{S}$ & 552 \\
Bilirubin & $\mathrm{U}, \mathrm{S}$ & 560 \\
Leucine aminopeptidase & $\mathrm{U}$ & 568 \\
Calcium & $\mathrm{U}, \mathrm{S}$ & 575 \\
Oxalate & $\mathrm{U}$ & 583 \\
Total protein & $\mathrm{U}$ & 591 \\
Albumin & $\mathrm{U}, \mathrm{S}$ & 600 \\
\hline
\end{tabular}

the biomolecule concentration. A light-to-frequency converter was integrated with the photodetectors to convert the analogue signal into a digital signal. Rather than just one optical filter [8], a 16 optical passband filter array has been developed, which allows quantifying the concentration of 16 different biomolecules with the same Microlab. The optical filters allow the use of only a white light source for illumination, giving portability to the Microlab.

Table 1 presents 16 biomolecules that can be analysed by optical absorption in a part of the visible spectrum. The second column indicates in which biological fluid the selected biomolecules can be analysed. The third column presents the wavelength at which each mixture has its maximum of absorbance. These values are very close $(8 \mathrm{~nm}$ between them, on average). Therefore, the optical passband filters must be highly selective; for example, their FWHM (full-width-halfmaximum) should be less than $6 \mathrm{~nm}$ to avoid misidentification in the analysis by possible overlapping absorbance spectra of mixtures. It should be noted that this problem is aggravated by the fact that the bandwidth of the absorption spectrum of for example uric acid extends over $70 \mathrm{~nm}$.

\subsection{Selection of the optical passband filter structure}

The optical filters should be designed to yield a narrow passband around the wavelength for which the coloured mixture being analysed has its absorption maximum (see table 1). This characteristic enables the selective measurement of the light intensity, at the desired wavelength, transmitted through the mixture.

Three approaches can be used for the optical filter structure: (1) a dielectric multilayer structure; (2) a FabryPerot structure with metallic mirrors and (3) a Fabry-Perot structure with dielectric mirrors. The best option in terms of optical characteristics, accuracy, feasibility, and fabrication process is the Fabry-Perot structure with dielectric mirrors. However, the first two have been previously published by the authors in [9] and [10], respectively. The major differences and the new contributions of the third approach described here are concerned with the spectral range that is fulfilled (from 480 to $600 \mathrm{~nm}$, as required in the third column of table 1), with the high selectivity achieved with low FWHM and with the simplicity and accuracy of the fabrication process (see section 5).

The Fabry-Perot optical filter consists of two parallel mirrors with a resonance cavity in the middle [11]. The equation $\lambda q=2 n d$ shows its operation principle, where $n$ is the refractive index of the cavity medium, $d$ the cavity length, $\lambda$ the incident wavelength and $q$ the interference order $(q=1,2,3, \ldots)$. Therefore, in a FabryPerot optical filter the thickness of the resonance cavity determines the tuned wavelength. The mirrors are dielectric mirrors, which, when properly designed and fabricated, offer high performance characteristics (high reflectivity with low absorption losses) [12]. These features enable the high selectivity required in the reported application.

\subsection{Selection of materials}

The dielectric mirrors of the Fabry-Perot optical filter are composed of a stack of $\mathrm{TiO}_{2}$ and $\mathrm{SiO}_{2}$ thin films (materials with high and low refractive index, in the visible spectrum, about 3.0 and 1.5, respectively). These dielectric films are also hard materials; thus it is extremely difficult, or even almost impossible, to remove them from the substrate. $\mathrm{SiO}_{2}$ has been selected because the wavelength dependence of its refractive index for the spectral band between 480 and $700 \mathrm{~nm}$ is almost constant (1.465 to 1.457 , respectively). $\mathrm{TiO}_{2}$ has been selected due to fabrication constraints (the deposition process is wellcharacterized).

\subsection{Optical simulations of the Fabry-Perot filters}

A thin-film optics software package (TFCalc 3.4) was used for the structural optimization of the optical filters. Simulation results show that a multilayer stack (five layers) of $\mathrm{TiO}_{2} / \mathrm{SiO}_{2}$ thin films for the dielectric mirrors and $\mathrm{a} \mathrm{SiO}_{2}$ layer for the cavity (see table 2) is the best option for the optical filters in terms of optical characteristics, feasibility and fabrication process. Figure 3 presents the simulated transmittances for all the 16 Fabry-Perot optical channels, with the layer stacks described in table 2. The $\mathrm{SiO}_{2}$ thickness of layer 6 changes from 140 to $230 \mathrm{~nm}$ in $6 \mathrm{~nm}$ steps. These results show that 
An array of highly selective Fabry-Perot optical channels for biological fluid analysis

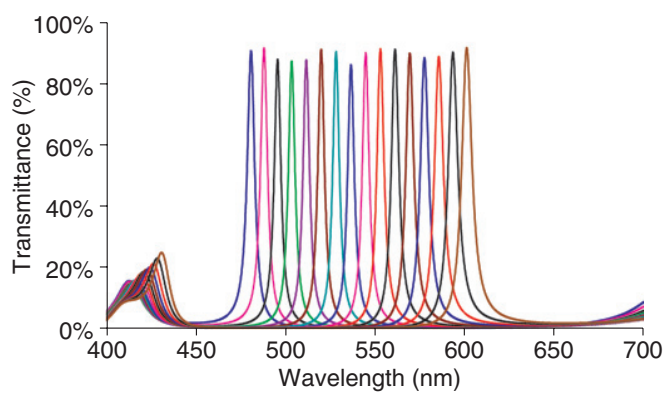

Figure 3. Simulated spectral transmittance for the 16 Fabry-Perot optical filter array, with the Fabry-Perot layer stacks described in table 2 .

each of the channels is sensitive to a single spectral band, with FWHM $<6 \mathrm{~nm}$. Furthermore, the ratio between the transmitted peak and the baseline is larger than 3.5 and the peaks transmittance is higher then $86 \%$. The filters are tuned to cover the 16 different spectral bands by adjusting the thickness of only the sixth layer, without affecting the Microlab layout. The performance of the optical filters could be increased by increasing the number of layers of the dielectric mirrors, but the fabrication process complexity would also increase. The commercially available passband optical filter on the top of the Microlab is used to avoid transmission of the non-visible part of the spectrum.

\subsection{Photodetectors}

Each optical channel is composed of a Fabry-Perot optical filter with a microfluidic channel and an optical detector underneath (see figure 2). The detector is an $n+/ p$-epilayer junction photodiode fabricated in a CMOS process. Since technology rules from the CMOS process have to be met, the design of the optical path is restricted to combinations of the three dielectric layers available above the pn-junction. Simulations show that the photodiode structure without two of those three dielectric layers provides the best possible quantum efficiency in the desired spectral range of photodiode structures available in a CMOS process [5]. Also, a dark current compensation channel is implemented using a photodiode completely covered with metal. Since the dark current is temperature dependent, one measurement at the beginning of the experiment would usually not be sufficient.

\section{Fabrication of the 16 optical channel array}

The Fabry-Perot optical filter array is deposited on top of the microchannels' glass die (see figure 1(a)). The three microchannels (A, B and $\mathbf{C}$ ) use the same set of filters. Films are deposited by IBD (ion beam deposition) from high-purity $\mathrm{Ti}$ and $\mathrm{SiO}_{2}$ targets $(99.90 \%$ and $99.99 \%$, respectively). The IBD system comprises an ion beam source integrated into a sputtering evaporator for pre-cleaning, in situ cleaning, etching and assisted deposition. The last one features high-quality thin-film fabrication. This technique presents the following advantages (over other deposition methods): good control of the deposition conditions and low deposition rates leading to good thickness uniformity; high adhesion; low porosity of the (a)

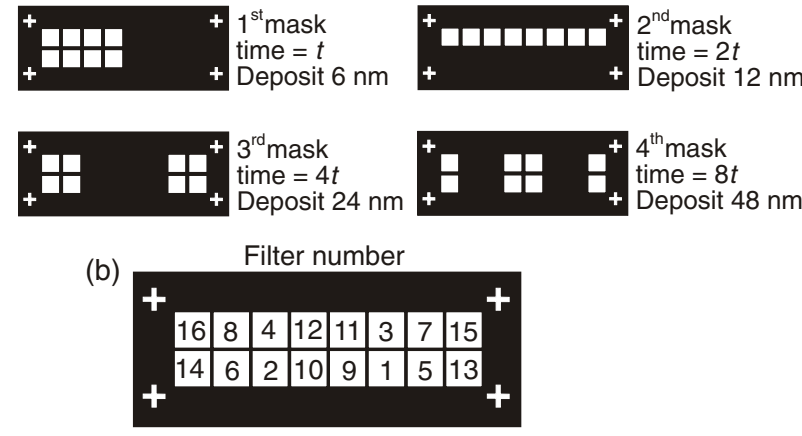

Figure 4. (a) The four masks used in the deposition process of the $\mathrm{SiO}_{2}$ layer 6. The crosses are alignment marks; (b) the position of each filter in the array.

films; low level of contamination; low film stress (it is a cold process). The relative deposition non-uniformity is $2 \%$ over an area of $10 \mathrm{~cm}^{2}$ [13].

The filter fabrication starts with the deposition of a $45 \mathrm{~nm}$ $\mathrm{TiO}_{2}$ layer over the entire array. Subsequently, layers 2-5 are deposited again over the entire array. Then, a $140 \mathrm{~nm}$ thick $\mathrm{SiO}_{2}$ layer is deposited over the entire array, which corresponds to the minimum thickness of layer 6 . In subsequent deposition steps, for which a mask is used and each of them with a different deposition time (see figure 4(a)), the total thickness of the $\mathrm{SiO}_{2}$ layer 6 increases from 140 to $230 \mathrm{~nm}$ in $6 \mathrm{~nm}$ steps, forming the filters numbered $1(\lambda=480 \mathrm{~nm})$ to 16 $(\lambda=600 \mathrm{~nm})$. Figure $4(\mathrm{~b})$ shows the position of each filter in the array. The formation of the 16 resonance cavities, each with a different thickness, requires further explanation (see figure 5). The first mask is used during a deposition time, $t$, for depositing $6 \mathrm{~nm}$ of $\mathrm{SiO}_{2}$, which results in two different thickness zones (see figure 5(a)). The second mask is used during a deposition time, $2 t$, for depositing $12 \mathrm{~nm}$ of $\mathrm{SiO}_{2}$, which results in four different thicknesses of layer 6 (see figure 5(b)). Therefore, each mask is used during a deposition time, $2^{(n-1)} t$ ( $n$ is the mask number), which results in $2^{n}$ different thicknesses of the $\mathrm{SiO}_{2}$ layer 6. The complete deposition sequence for layer 6 is shown in figure 5. A relative thickness uniformity better than $1 \mathrm{~nm}$ was achieved between cavity lengths. After completion of the 16 different thicknesses of layer 6 , the top mirror formed by layers 7-11 is deposited over the entire array. An SEM (secondary electron microscopic) photograph presenting the cross-section of one of the channels (filter number 3 ) is shown in figure 6 . The 16 Fabry-Perot optical filter array fabrication requires only 4 masks and 15 deposition steps. The optical filters can be easily tuned to different spectral bands by adjusting only the thickness of layer 6, without affecting the Microlab layout.

Figure 7 shows a photograph of the Microlab. The holes are drilled and the microchannels are milled in glass wafers and then both wafers are bonded. The CMOS compatible photodetectors and readout circuits are fabricated through a single-polysilicon, double-metal, $1.6 \mu \mathrm{m}$ n-well CMOS process. The area of each optical channel is $200 \times 200 \mu \mathrm{m}^{2}$. The die area is $2.0 \times 5.3 \mathrm{~mm}^{2}$. The glass die is glued to the CMOS chip. 
(a)

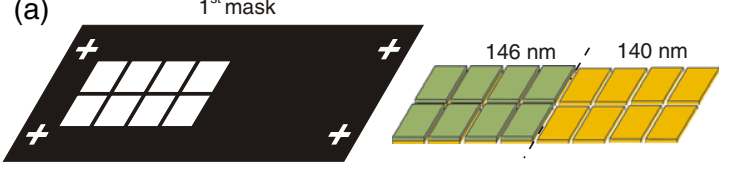

(c)

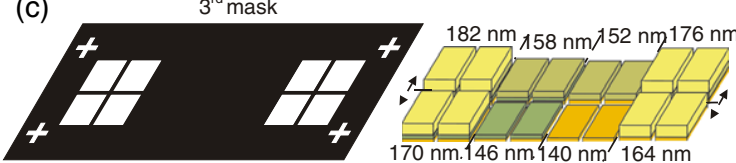

(b) $\quad 2^{\text {nd }}$ mask
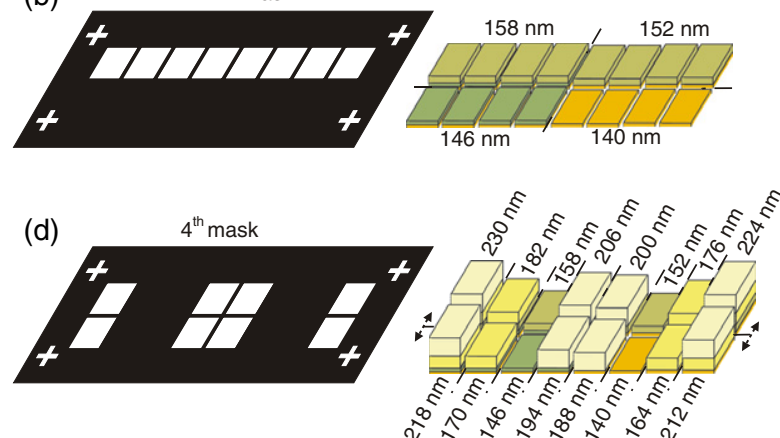

Figure 5. The deposition process of the $\mathrm{SiO}_{2}$ layer 6. (a) Applying the first mask, two different $\mathrm{SiO}_{2}$ thickness are obtained; (b) applying the second mask, four different $\mathrm{SiO}_{2}$ thickness are obtained; (c) applying the third mask, eight different $\mathrm{SiO}_{2}$ thickness are obtained; (d) applying the fourth mask all the $16 \mathrm{SiO}_{2}$ thickness are obtained.

Table 2. Layer materials and thicknesses for the 16 Fabry-Perot optical filter array.

\begin{tabular}{|c|c|c|c|c|c|c|c|c|c|c|c|c|c|c|c|c|c|}
\hline \multicolumn{2}{|c|}{ Filter number } & 1 & 2 & 3 & 4 & 5 & 6 & 7 & 8 & 9 & 10 & 11 & 12 & 13 & 14 & 15 & 16 \\
\hline & Peak $(n m)$ & 480 & 488 & 495 & 503 & 512 & 520 & 528 & 536 & 544 & 552 & 560 & 568 & 575 & 583 & 591 & 600 \\
\hline Layer & Material & \multicolumn{16}{|c|}{ Thickness of each layer (nm) } \\
\hline 1 & $\mathrm{TiO}_{2}$ & 45 & 45 & 45 & 45 & 45 & 45 & 45 & 45 & 45 & 45 & 45 & 45 & 45 & 45 & 45 & 45 \\
\hline 2 & $\mathrm{SiO}_{2}$ & 95 & 95 & 95 & 95 & 95 & 95 & 95 & 95 & 95 & 95 & 95 & 95 & 95 & 95 & 95 & 95 \\
\hline 3 & $\mathrm{TiO}_{2}$ & 45 & 45 & 45 & 45 & 45 & 45 & 45 & 45 & 45 & 45 & 45 & 45 & 45 & 45 & 45 & 45 \\
\hline 4 & $\mathrm{SiO}_{2}$ & 95 & 95 & 95 & 95 & 95 & 95 & 95 & 95 & 95 & 95 & 95 & 95 & 95 & 95 & 95 & 95 \\
\hline 5 & $\mathrm{TiO}_{2}$ & 45 & 45 & 45 & 45 & 45 & 45 & 45 & 45 & 45 & 45 & 45 & 45 & 45 & 45 & 45 & 45 \\
\hline 6 & $\mathrm{SiO}_{2}$ & 140 & 146 & 152 & 158 & 164 & 170 & 176 & 182 & 188 & 194 & 200 & 206 & 212 & 218 & 224 & 230 \\
\hline 7 & $\mathrm{TiO}_{2}$ & 45 & 45 & 45 & 45 & 45 & 45 & 45 & 45 & 45 & 45 & 45 & 45 & 45 & 45 & 45 & 45 \\
\hline 8 & $\mathrm{SiO}_{2}$ & 95 & 95 & 95 & 95 & 95 & 95 & 95 & 95 & 95 & 95 & 95 & 95 & 95 & 95 & 95 & 95 \\
\hline 9 & $\mathrm{TiO}_{2}$ & 45 & 45 & 45 & 45 & 45 & 45 & 45 & 45 & 45 & 45 & 45 & 45 & 45 & 45 & 45 & 45 \\
\hline 10 & $\mathrm{SiO}_{2}$ & 95 & 95 & 95 & 95 & 95 & 95 & 95 & 95 & 95 & 95 & 95 & 95 & 95 & 95 & 95 & 95 \\
\hline 11 & $\mathrm{TiO}_{2}$ & 45 & 45 & 45 & 45 & 45 & 45 & 45 & 45 & 45 & 45 & 45 & 45 & 45 & 45 & 45 & 45 \\
\hline
\end{tabular}

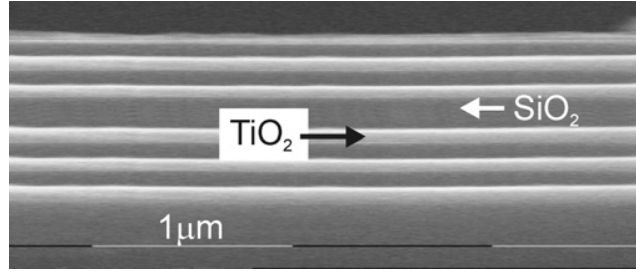

Figure 6. SEM photograph showing the cross-section of the Fabry-Perot optical filter number 3 (according to table 2).

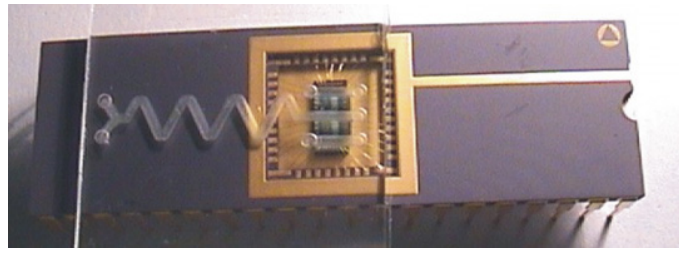

Figure 7. Photograph of the Microlab. The Fabry-Perot optical filter array has only a slight colouring (invisible in the photograph).

\section{Experimental results}

The electrical characteristics and the spectral responsivity were measured using a Keithley 487 picoamperemeter (full- scale range from $10 \mathrm{fA}$ to $2 \mathrm{~mA}$ and a resolution of $5^{1 / 2}$ digit), and a $250 \mathrm{~W}$ quartz tungsten halogen lamp with the ORIEL Cornerstone $130^{\mathrm{TM}}$ monochromator used as light source. The on-chip photodiodes were calibrated with a calibrated commercially available photodiode as reference (Hamamatsu S1336-5BQ). The electrical characterization of the detection and readout circuits were presented and a detailed explanation given in [8]. The photodiode dark current is $1.08 \times$

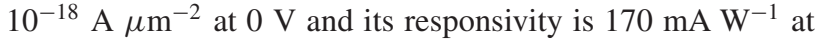
$\lambda=495 \mathrm{~nm}$.

\subsection{Opto-electric performance of the Microlab}

The opto-electric performance is a trade-off between sensitivity, selectivity and number of optical channels used. Figure 8 presents the measured spectral responsivity for all 16 optical channels. The ratio between the baseline and the peak maximum ranges from 3.7 to 10 . The FWHM ranges from 3.5 to $7 \mathrm{~nm}$. This optical spectra measurements show that each of the optical channels is sensitive to only one narrow spectral band centred at the wavelength for which the biomolecule being analysed has its absorption maximum. Variations (comparing with figure 3 ) in the height of the peaks are due, mainly, to the spectral sensitivity of the integrated photodiodes. Moreover, variations in the peak transmitted wavelength are about $0.9 \mathrm{~nm}$. They are due to a $0.2 \%$ error in the total thickness 
An array of highly selective Fabry-Perot optical channels for biological fluid analysis

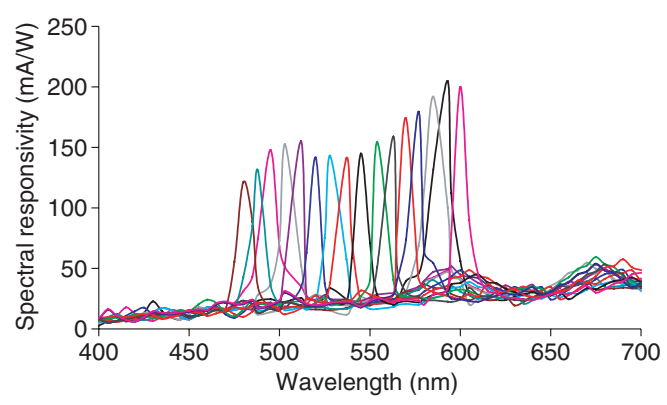

Figure 8. Measured spectral responsivity of all optical channels (optical effect of photodiode and optical filter included).

of the Fabry-Perot optical filters, when compared to the designed thickness. However, this small error will not affect the final application of the Microlab, once the biomolecule detection is performed by measuring simultaneously the light transmitted through the three microchannels (A, B and C, e.g., relative measurements). Any imperfection of the incident light wave and the roughness surface of the Fabry-Perot optical filter are both responsible for the increased transmittance outside the narrow band to which the Fabry-Perot resonance cavity is tuned (the background signal). In addition, a compensation structure with the cavity optical length below $\lambda / 10$ should be used. The transmittance signal of this structure is similar to the parasitic background and it is subtracted from the real signal [14]. It should be mentioned that the conventionally used dark current photodiode compensates only for the nonidealities of the detector itself. This method compensates for the nonidealities of the detector, Fabry-Perot optical filter and incident light, at the same time.

\subsection{Quantitative measurement of uric acid in the Microlab}

The Microlab operation is demonstrated in the spectrophotometric analysis of uric acid in urine, especially in the quantitative measurement of its concentration. The reagent used in the measurements is the Infinity ${ }^{\mathrm{TM}}$ Uric Acid Reagent from SigmaAldrich. This reagent reacts with a sample of urine containing uric acid in a 50:1 ratio, and produces an absorption maximum at a specific wavelength $[6,15]$. Figure 9 shows the measured transmittance response through the entire optical channel for different uric acid concentrations (up to $120 \mathrm{mg} \mathrm{dl}^{-1}$ ). The transmittance is defined as $T=I / I_{0}$, where $I$ is the measured photodiode current for each mixture and $I_{0}$ the measured photodiode current of the reagent. The optical lightpath is $500 \mu \mathrm{m}$ (the depth of the microchannels). The reproducibility mean coefficient variation of ten replicate measurements for each concentration is less than $10 \%$. The transmittance at $\lambda=495 \mathrm{~nm}$ as a function of the uric acid concentrations is shown in figure 10. Data from the linear regression of the transmittance calibration plot give a slope of $(-4.04 \pm 0.22) \times 10^{-3}$ and an intercept of $0.87 \pm 3.71 \times 10^{-3}$. With a correlation coefficient $\left(R^{2}\right)$ of 0.988 , the fit can be considered linear for concentrations as large as $40 \mathrm{mg} \mathrm{dl}^{-1}$. For higher concentrations the sample should be diluted and re-assayed, multiplying the result by the dilution factor.

The presence of the Fabry-Perot optical filter limits the light transmitted to the underlying photodiode (the maximum

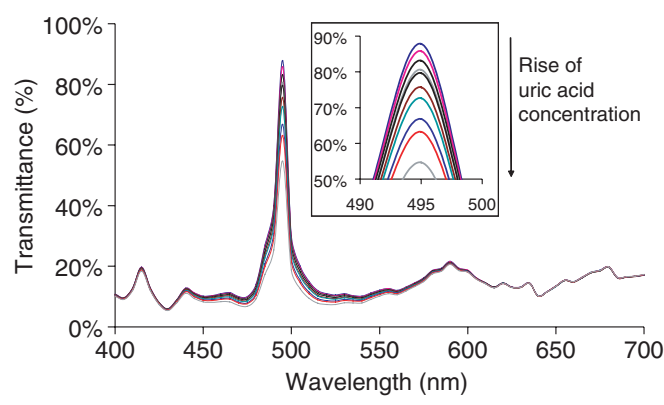

Figure 9. Measured transmittance spectra for different uric acid concentrations (optical effect of photodiode and optical filter 3 included).

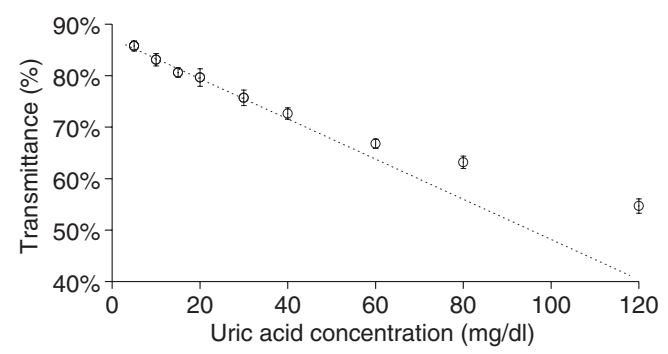

Figure 10. Calibration curve at $\lambda=495 \mathrm{~nm}$ (mean value \pm standard deviation).

transmittance value of the reagent deceases from $100 \%$ to the maximum spectral transmittance of the corresponding filter, $88 \%$ for the filter number 3 ). However, once the FabryPerot optical filter is composed of high-performance dielectric mirrors, the light transmitted through the optical filter is more than $85 \%$ of the incident light.

Design, simulations and measurements for the same uric acid concentration were previously performed with two different optical filters: an array of Fabry-Perot filters with metallic mirrors [10], and an array of a dielectric multilayer structure (without a Fabry-Perot structure) [9]. In both, the spectral range from 480 to $600 \mathrm{~nm}$ was not fulfilled. Moreover, the maximum transmittance through the FabryPerot optical filter with metallic mirrors was less than $10 \%$, since metallic mirrors cannot provide both high transmittance and low FWHM. They are attractive due to the simplicity of their fabrication; only three layers are deposited and the fabrication requires only four masks and six deposition steps for an array of 16 optical channels. The optical filters with a dielectric multilayer structure featured high transmittance and low FWHM. However, the fabrication of the complete structure is significantly more complex. It needs 34 masked deposition steps and four masks that must be rotated and vertically flipped, for the same 16 optical channels. The fabrication of the 16 optical channels reported here requires only four masks and 15 deposition steps.

The spectral measurements of figures 8 and 9, when compared with the ones without the optical filter [5], confirm that it can be used with a white light source for the Microlab illumination, which highly facilitates portability and measuring with instantaneous results at low cost. Moreover, the uric acid concentration was measured while illuminating the 


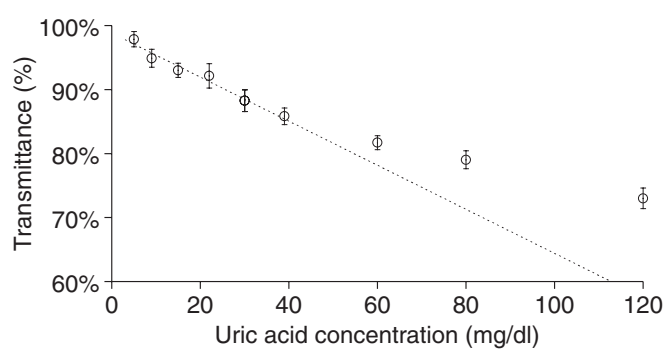

Figure 11. Uric acid concentration measurement using only a white light source ( $200 \mathrm{~W}$ halogen lamp) for illuminating the Microlab (mean value \pm standard deviation).

Microlab with a white light source (see figure 11). The transmittance value of $100 \%$ corresponds to the measured transmittance of the reagent (microchannel A). The resultant fit parameters with a slope of $(-3.70 \pm 0.37) \times 10^{-3}$, an intercept of $0.99 \pm 6.21 \times 10^{-3}$ and a correlation coefficient of 0.962 confirm that the fit can still be considered linear (up to $40 \mathrm{mg} \mathrm{dl}^{-1}$ ). Therefore, the measurements confirm the proportional dependence between intensity of the colour produced by the mixture and the biomolecule concentration. Moreover, any white light source can be used for illumination, since the Microlab compensates the light fluctuations by measuring the photocurrent of the three photodiodes under the three microchannels simultaneously. Readout electronics integrated with the photodiodes combines data, makes decisions and provides a standardized output format for computer levels. The minimum detection of the Microlab is $0.5 \mathrm{mg} \mathrm{dl}^{-1}$ and the achieved sensitivity is $5 \mathrm{mg} \mathrm{dl}^{-1}$, which corresponds to a relative resolution of $3.3 \%$, enough for human being urine values. These results agree with macroscopic measurements performed with wellknown uric acid standards and using state-of-the-art laboratory equipment [5].

Higher sensitivity could be obtained if, instead of the optical absorption technique, other optical sensing techniques had been used, such as fluorescence, refractometry or surface plasmon resonance, for example. However, the optical detection microsystem will need a more expensive readout optics, which for the target application of the Microlab, usually called 'routine analyses', would not be necessary, since the achieved sensitivity is enough and, as said, the obtained results agree with those of the state-of-the-art clinical laboratory equipment (this laboratory equipment uses the optical absorption technique).

\section{Conclusions}

The optical Microlab presented here offers unique opportunities for spectrophotometric analysis in clinical diagnostics, in addition to the general advantages of a laboratory microsystem concerning small sample volume, which is the use of white light illumination made possible by the integration of 16 highly selective optical filters, based on Fabry-Perot thin-film optical resonators. This feature highly facilitated portability and measuring with instantaneous results at low cost. The Microlab allows measuring the concentration of 16 different biomolecules in biological fluids with the same device. Its performance was successfully demonstrated in the quantitative measurement of uric acid in a human's urine. However, other molecules or biological fluids are potential candidates for the Microlab. Moreover, the number of analysed biomolecules can be expanded by fabricating 32 optical channels, using the same fabrication principles. In this case, an additional fifth mask and deposition step (time $=16 t$ ) would be needed for the resonance cavities. This technique can be extended further to yield a device with 64 or 128 optical channels. The Microlab's highly selective filtering system makes the device broadly applicable and a powerful tool in hospitals, operating rooms, doctors' offices, and clinical laboratories as well as in patients' homes.

\section{Acknowledgments}

The authors wish to acknowledge Ger de Graaf, from the Laboratory for Electronic Instrumentation, TUDelft, The Netherlands, for his help with device fabrication. They also wish to acknowledge Paulo Freitas and Susana Freitas, from the INESC-MN, Portugal, for filter fabrication.

\section{References}

[1] Connolly P 1995 Clinical diagnostics opportunities for biosensors and bioelectronics Biosensors Bioelectron. $101-6$

[2] Strasinger S K and Di Lorenzo M S 2001 Urianalysis and Body Fluids 4th edn (Philadelphia, PA: F A Davis Company)

[3] http://www.hypoguard.com/diascreen_reagent_strips.html

[4] Hahn J H, Ro K W, Shim B C and Lim K 2003 Absorbance detection system for lab-on-a-chip, US2003/0017079A1

[5] Minas G, Martins J S, Ribeiro J C, Wolffenbuttel R F and Correia J H 2004 Biological microsystem for measuring uric acid in biological fluids Sensors Actuators A $11033-8$

[6] Biochemical and Organic Reagents 2002 Sigma-Aldrich

[7] Minas G, Wolffenbuttel R F and Correia J H 2005 Lab Chip 5 1303-9

[8] Minas G, Ribeiro J C, de Graaf G, Wolffenbuttel R F and Correia J H 2004 Proc. IEEE Sensors 2004 (Vienna, Austria) pp 223-6

[9] Minas G, Ribeiro J C, Lanceros-Mendez S, Vaz F, Wolffenbuttel R F and Correia J H 2003 Proc. Transducers'03 (Boston, USA) pp 1251-4

[10] Minas G, Martins J S, Ribeiro J C, Wolffenbuttel R F and Correia J H 2004 Sensors Actuators A 115 362-7

[11] Saleh B and Teich M C 1991 Fundamentals of Photonics (New York: Wiley)

[12] Macleod H A 2001 Thin-Film Optical Filters 3rd edn (Bristol: Institute of Physics Publishing)

[13] http://www.inesc-mn.pt/listequip.htm

[14] Bartek M, Correia J H, Kong S H and Wolffenbuttel R F 1999 Proc. Transducers'99 pp 240-3

[15] Kabasakalian P, Kalliney S and Westcott A 1973 Determination of uric acid in serum, with use of uricase and a tribromophenol-aminoantipyrine chromogen Clin. Chem. 19 $522-4$ 